\title{
How does the GRS1915+105 plateau state compare to the canonical hard state?
}

\author{
P.C.N. van Oers, Sera Markoff, Dipankar Maitra \\ Astronomical Institute "A. Pannekoek", University of Amsterdam, Kruislaan 403, 1098 SJ, The \\ Netherlands \\ E-mail: poers@science.uva.nI
}

Michael Nowak

Kavli Institute for Astrophysics and Space Research, Massachusetts Institute of Technolgy, Cambridge, USA

\section{Jörn Wilms}

Dr. Karl Remeis-Sternwarte, Astronomisches Institut, Universität Erlangen-Nuremberg, Sternwartstr. 7, 96049, Germany

\section{Alberto Castro-Tirado}

Instituto de Astrofisica de Andalucia (IAA-CSIC), 18008 Granada, Spain

\section{Emilios Harlaftis * \\ Jerome Rodriguez}

Laboratoire AIM, CEA/DSM-CNRS-Université Paris Diderot, DAPNIA/SAp, F-91191 Gif sur Yvette, France

\section{Vivek Dhawan}

National Radio Astronomy Observatory, 10003 Lopezville Road, Socorrow, NM 87801, USA

\begin{abstract}
GRS1915+105 is a very peculiar black hole binary (BHB), as it presents us with some states never before seen in other black hole systems. However one of these states, referred to as the plateau or $\chi$ state, may be related to the canonical hard state because of its association with steady, relatively lower X-ray emission and flat/inverted radio emission. Compact, self-absorbed jets have also been observed in this state. In order to investigate the relationship between the plateau and the hard state, we fit two multi-wavelength observations using a steady-state outflowdominated model, developed for hard state BHBs. The data sets consist of quasi-simultaneous observations in radio, IR and X-ray. Interestingly, we find both significant differences between the two plateau states, as well as between the best-fit model parameters and those representative of the hard state. We discuss our interpretation of these results, and the possible implications for GRS1915+105's relationship to canonical black hole candidates.
\end{abstract}

VII Microquasar Workshop: Microquasars and Beyond September 1-5 2008

Foca, Izmir, Turkey

\footnotetext{
* Deceased
} 


\section{Introduction}

The microquasar GRS1915+105 was discovered on the 15th of August 1992, by the WATCH all-sky monitor, aboard the Russian GRANAT satellite. It is an hard X-ray transient located in the constellation of Aquila, at $l=45.37^{\circ}, b=-0.22^{\circ}$. GRS1915+105 is well-known for its remarkable variability in the X-ray band, distinguishing it from every other X-ray source known.

Belloni et al. (2000) were able to classify data stretching over more then a year into twelve classes, based on their color-color diagram and light curves. Most classes are understood as the interplay of basic states (A, B and C). Class $\chi$ is reserved for state $\mathrm{C}$ exclusively and is usually called the plateau state. As both are associated with outflows (Dhawan et al. 2000, McClintock \& Remillard 2003), it is tempting to compare the plateau state to the canonical hard state (HS). The average luminosity is however a factor 100 higher and the X-ray photon index is usually steeper (McClintock \& Remillard 2003) during a plateau. Reig et al. (2003) argue that the HS is really never seen in GRS1915+105 as a power-law tail is always present.

In this work we compare the results of best-fit models to two multi-wavelength plateau state observations of GRS1915+105 to HS observations of GX339-4, GRO J1655-40 and XTE1118+480.

\section{Observation}

Based on the Hardness Intensity Diagrams (HID) (figure 1), lightcurves and spectral index (figure 2), GRS1915+105 was in the plateau state on MJD 51367 (radio quieter) and MJD 53473 (radio louder). To obtain multi-wavelength spectra for these episodes, quasi-simultaneous radio and infrared data were added to X-ray data, taken with RXTE (table 2).

\section{Model / Physical Parameters}

All fits were done using a steady-state outflow-dominated model (Markoff, Nowak \& Wilms 2005) with a gaussian at $\sim 6.4 \mathrm{keV}$, a blackbody to model the companion star in the infrared and a multi color blackbody to model the disk. To these, two other models were added: Compton reflection from a neutral medium (reflect) and the smeared edge model (smedge) (by Magdziarz, Zdziarski 1995 and Ebisawa 1991 respectively). When possible, we froze free parameters, based on literature (table 3).

\section{Results}

The best fits obtained using our model are in figure 4. As we used several combinations of

\begin{tabular}{l|lllll||llll|} 
& \multicolumn{5}{|c||}{ Dataset A 1999 Juli 8 / MJD 51367 } & \multicolumn{3}{c|}{ Dataset B 2005 April 13 / MJD 53473 } \\
\hline Band & Instrument & MJD start & UTC & Duration & Instrument & MJD start & Time & Duration \\
\hline X-ray & RXTE & & 51367.2737 & $06: 34: 13$ & $14435 \mathrm{sec}$ & RXTE & 53473.054 & $01: 09: 05$ & $6769 \mathrm{sec}$ \\
IR & UKIRT & $(J)$ & 51367.5753 & $13: 48: 27$ & $64 \mathrm{~min}$ & CTIO $(K)$ & 53472.3216 & $07: 43: 06$ & $25 \mathrm{~min}$ \\
& & $(H)$ & 51367.4821 & $11: 34: 10$ & $80 \mathrm{~min}$ & & & & \\
& & $(K)$ & 51367.8216 & $07: 56: 59$ & $64 \mathrm{~min}$ & & & & \\
Radio & GBI & & 51367.371 & $09: 02: 53$ & $30 \mathrm{sec}$ & Ryle & 53473.098 & $02: 21: 07$ & $1 \mathrm{sec}$ \\
\hline
\end{tabular}

Table 1: Observations included in dataset A and B. The RXTE ObsId's are 40403-01-09-00 and 90105-0702-00 respectively. The $J, H$ and $K$ bands are at 1.002-1.322, 1.455-2.094 and 1.906-2.547 $\mu \mathrm{m}$, respectively. The $K$ band observation was done (more than half a day earlier) with the Andicam at CTIO. The GBI measurements are $2.25 \& 8.3 \mathrm{GHz}$. The Ryle telescope operates at $15 \mathrm{GHz}$ and the flux density used is the average of a 03:38:53 long observation. 


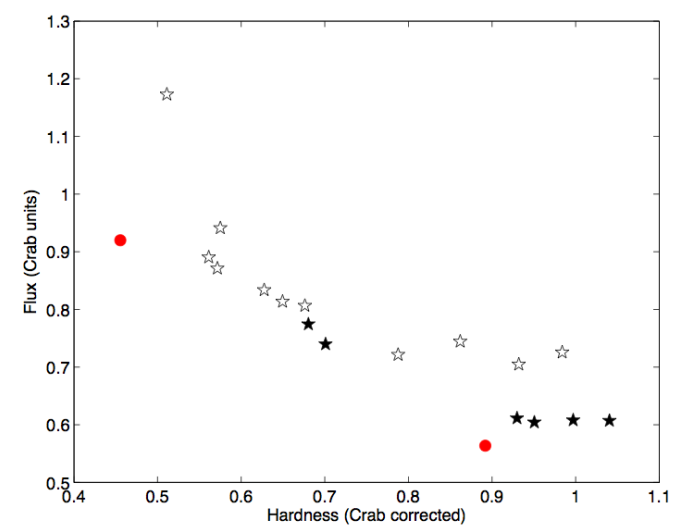

Figure 1: HID comparing our observations (red dots) to the plateau states from Belloni et al. 2000 (stars). The soft, higher-luminosity red dot is ObsID 90105-07-02-00, the other one is 40403-01-09-00. (Courtesy of T. Belloni)
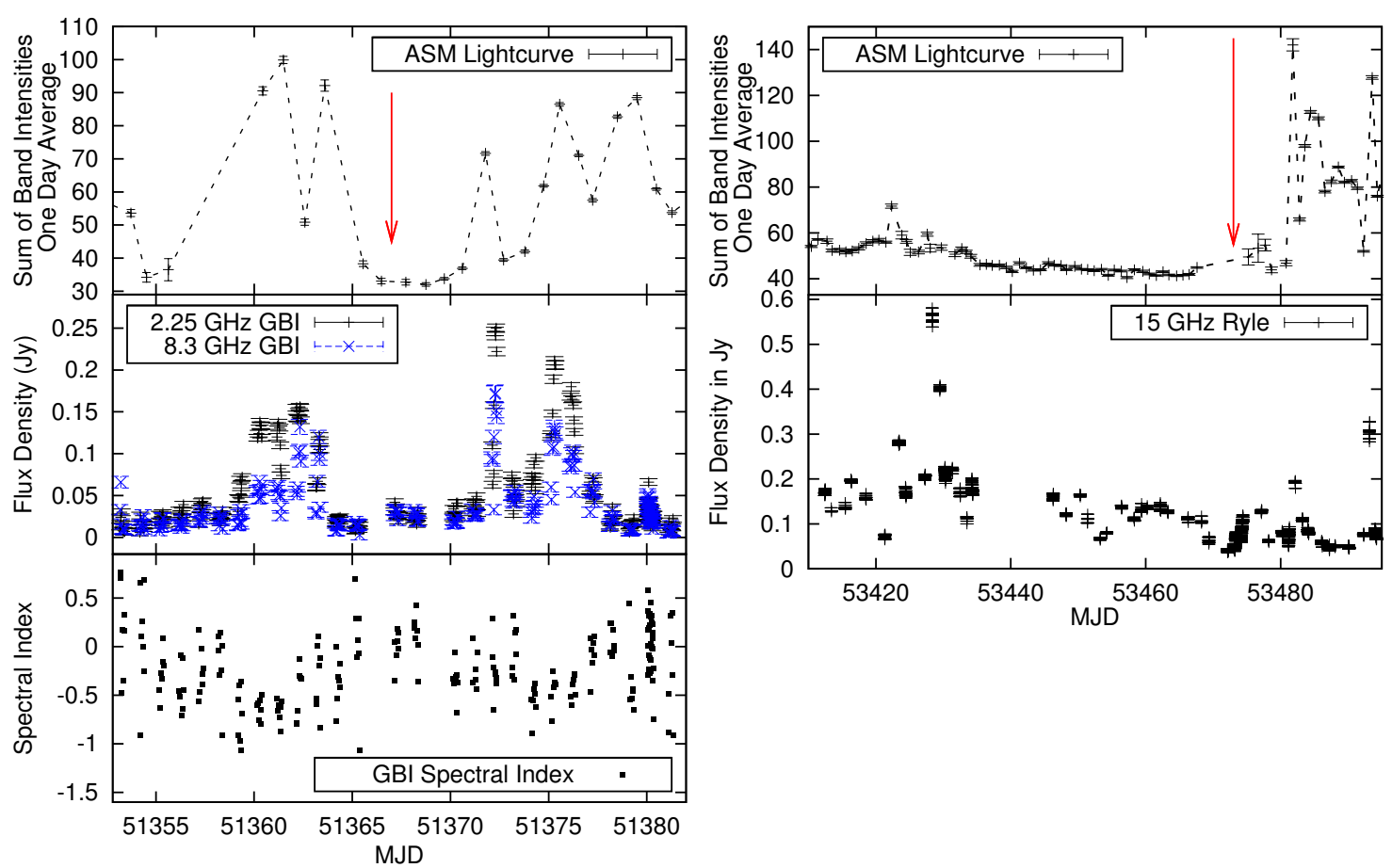

Figure 2: GRS1915+105 X-ray and radio data for the 1999 (left) and 2005 (right) data set. The radio arrow indicates the plateau states.

\begin{tabular}{lrr}
\hline parameter & value & units \\
\hline column density & $4.7 \pm 0.2$ & $10^{22} \mathrm{~cm}^{-2}$ \\
mass & $14 \pm 4$ & $\mathrm{M}_{\odot}$ \\
inclination & $66 \pm 2$ & $\circ$ \\
distance & $11_{-5}^{+3}$ & $\mathrm{kpc}$ \\
donor temperature & $4455 \pm 190$ & $\mathrm{~K}$ \\
\hline
\end{tabular}

Table 2: Invariant parameters used in the fitting process. 

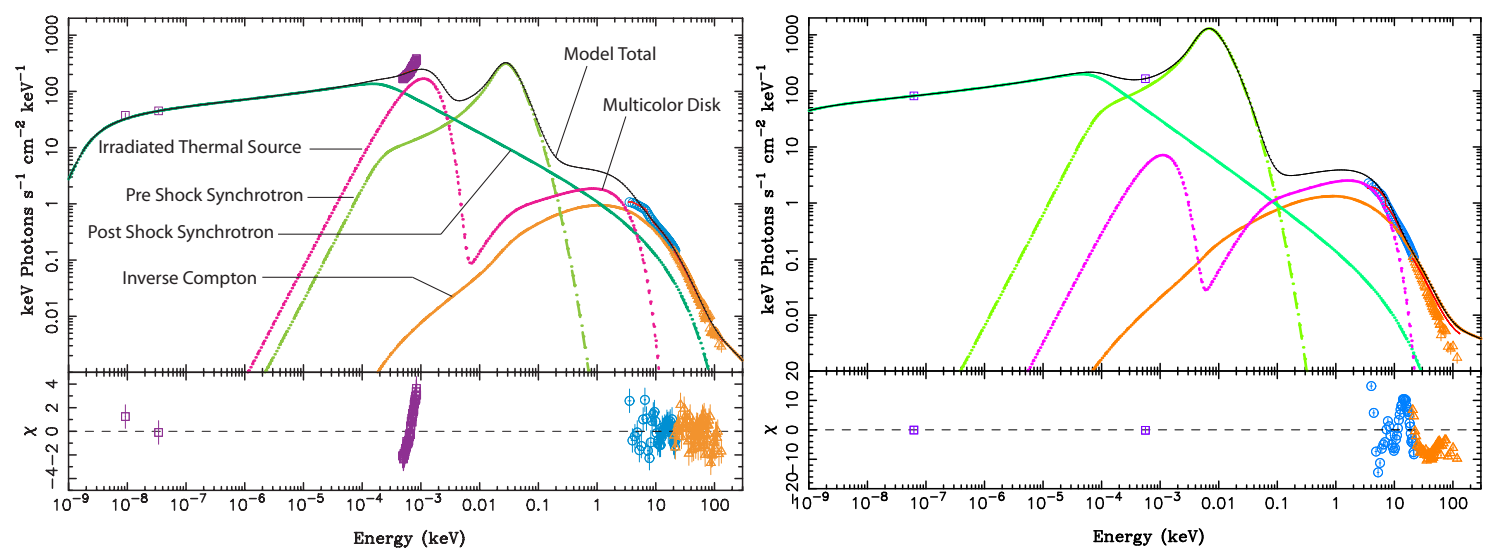

Figure 3: Results from fitting dataset A (left, $\chi_{\text {red }}^{2} \sim 1.8$ ) and dataset B (right, $\chi_{\text {red }}^{2} \sim 60$ ) with the outflow dominated, steady state model. The components (see left plot for legend) have not been forward folded through the detector response matrix and can therefore deviate from the model total output. For dataset A we only use a simple blackbody to model the companion star in the infrared. As the infrared data obviously displays much more structure and complexity the fit here is not good.

\begin{tabular}{lcccc} 
& & range found in & \multicolumn{2}{c}{ GRS1915+105 } \\
variable & units & canonical BHs & Dataset A & Dataset B \\
\hline$N_{j}$ & $10^{-3} \mathrm{~L}_{\text {Edd }}$ & $0.34-71$ & $505-837$ & $676-1305$ \\
$r_{0}$ & $G M / c^{2}$ & $3.5-20.2$ & $64.4-65.5$ & $80-202$ \\
$T_{e}$ & $10^{10} \mathrm{~K}$ & $2.0-5.23$ & $1.6-2.3$ & $2.35-2.40$ \\
$p$ & & $2.1-2.9$ & 2.1 & 2.5 \\
$k$ & & $1.1-7$ & $2.5-17$ & $2.6-14$ \\
$z_{a c c}$ & $G M / c^{2}$ & $7-400$ & $2400-2500$ & 15000 \\
$f_{\text {sc }}$ & $10^{-3}$ & $0.16-29.9$ & $0.17-0.21$ & $0.15-0.19$ \\
$\mathrm{~L}_{\text {disk }}$ & $10^{-3} \mathrm{~L}_{\text {Edd }}$ & $0.07-99$ & $62-97$ & $200-235$ \\
$T_{\text {disk }}$ & $\mathrm{keV}$ & $0.06-1.53$ & $0.7-1.5$ & $1.73-1.96$ \\
reflect & & $0-0.21$ & $0-0.26$ & 0 \\
\hline
\end{tabular}

Table 3: Parameter ranges found in canonical black holes and GRS1915+105. $N_{j}$ is the jet normalization, $r_{0}$ the nozzle radius, $T_{e}$ the temperature of the leptons as entering the jet, $p$ the spectral index of the radiating particles (fixed at stated values), $k$ the ratio between magnetic and electron energy densities, $z_{a c c}$ the distance of the particle acceleration region and $f_{\text {scat }} 0.36$ / ratio between scattering mean free path and gyro-radius.

models, we found ranges for the fitted parameters (table 4). We obtained satisfactory statistics on dataset A. For dataset B statistics are not sufficient to make any strong statements (see discussion). We will therefore only treat these results as an indication of a trend and base most of our conclusions on dataset $\mathrm{A}$.

\section{Discussion and Conclusions}

The jet model that was successfully used to model more canonical sources in the HS, also works at the high luminosity levels of the plateau state. It does however produce an entirely different regime in parameter space. For the radio louder state the results are inconclusive:

\subsection{Problems fitting MJD 53473}

In dataset A we were successfully able to use the Compton slope to fit the steep X-ray data. However for dataset B even the Compton slope is too shallow. Fitting with the synchrotron cutoff yields far better fits, but also brings along physical uncertainties. Trying to employ the exponential 
decay of the multicolor disk also fails because a higher disk contribution offers more (soft) seed photons. These photons are up-scattered and create a Compton tail in the hardest part of the spectrum, pushing the model flux higher then the observation. Possibly the model breaks down as the luminosity is close to, or even super-Eddington(see fig 1 and table 4).

\subsection{Comparing both data sets with the HS}

- Our luminosity $\left(N_{j}\right)$ is one to three magnitudes larger. Canonical black holes would have quenched their jets long before this luminosity was achieved.

- Looking at the radius of the base of the jet $r_{0}$ and the location of the jet shock wave $z_{a c c}$, the physical dimensions are much bigger. This size appears to be directly linked to its luminosity and is inferred from the mass (table 3).

- Also $f_{\text {sc }}$ is quite low, moving the synchrotron cut off to lower frequencies. For dataset $\mathrm{A}$ the Comptonization can dominate the harder X-rays allowing a fit for the extremely steep x-ray data in the plateau state.

- The particle distribution index $p$ was frozen to a low 2.1 for dataset A, contradicting the usual steeper power law. Higher values required an unrealistic power input into the jet. Lower values amounted to a fitting of the X-ray spectrum with the synchrotron cutoff. While McClintock \& Remillard (2003) found the photon index in the plateau state to be steeper, our values are within the usual range for the HS and the Quiescent State (Remillard \& McClintock 2006). The latter state is contradicted by the high $N_{j}$.

\subsection{Comparing our two plateaux to each other}

The disk contribution is much higher in dataset B. This is true for the luminosity as well as the temperature, while for the old dataset these are comparable to the canonical black holes. This confirms that, as for the HS, the classification of the plateau should not be based on luminosity.

\subsection{The Future}

Perhaps in the near future GRS1915+105 will retreat in its version of quiescence. This would further our insight into the behavior under lower luminosity conditions, where jets are thought to persist. The relation of the canonical Hard State to the $\chi$ state will be much better understood once we can compare them to other observations showing steady radio emission.

At this moment we are still exploring parameter space for both datasets. A more detailed discussion will be featured in van Oers et al. (in prep.).

\section{References}

[1] Belloni, T., Klein-Wolt, M., Méndez, M., et al, A\&A, 355, 271, 2000

[2] Dhawan, V., Mirabel, I.F., Rodríguez, L.F., ApJ, 543, 373-385, 2000

[3] Ebisawa, K., PhD thesis, 1991

[4] Magdziarz, P., Zdziarski, A.A., MNRAS, 273, 837, 1995

[5] Markoff, S.B., Nowak, M.A., Wilms, J., ApJ 635, 1203M, 2005

[6] McClintock, J.E., Remillard, R.A., ArXiv e-prints, 0306213, 2003

[7] Reig, P., Belloni, T., van der Klis, M., A\&A, 12, 229, 2003

[8] Remillard, R.A., McClintock, J.E., ARA\&A, 44, 49, 2006 